\title{
Stevens-Johnson Syndrome and Toxic Epidermal Necrolysis in Patients Admitted to Sina Hospital in Hamadan, Iran: A 16-year study
}

\author{
Pedram Alirezaei ${ }^{1}$, Mohammad Reza Sobhan$^{1}$, Ali Saadatmand ${ }^{2, *}$ \\ ${ }^{1}$ Assistant Professor of Dermatology, Psoriasis Research Center, Hamadan University of Medical Sciences, Hamadan, Iran \\ ${ }^{2}$ MSc in Microbiology, Psoriasis Research Center, Hamadan University of Medical Sciences, Hamadan, Iran
}

* Corresponding Author: Ali Saadatmand, Psoriasis Research Center, Hamadan University of Medical Sciences, Hamadan, Iran.Email: Ali.s_umsha@yahoo.com

Received: 24.09 .2018

Accepted: 17.01.2019

How to Cite this Article:

Alirezaei P, Sobhan MR, Saadatmand A. Stevens-Johnson Syndrome and Toxic Epidermal Necrolysis in Patients Admitted to Sina Hospital in Hamadan, Iran: A 16-year study. Avicenna J Clin Med. 2019; 25(4): 193199. DOI: $10.21859 /$ ajcm.25.4.

\section{Abstract}

Background and Objective: Stevens-Johnson syndrome (SJS) and toxic epidermal necrolysis (TEN) are severe and potentially life-threatening reactions. In spite of the low prevalence of these conditions, they are of utmost significance due to their catastrophic complications and mortality. The SJS and TEN are mostly caused by a reaction to a drug; however, in some cases, they result from infections. The aim of this study was to investigate the epidemiologic, etiologic, and clinical characteristics of the patients admitted to Sina Hospital in Hamadan, Iran, due to SJS and TEN during a 16 -year period.

Materials and Methods: This cross-sectional descriptive study was conducted on the patients with SJS and TEN admitted to Sina Hospital during the last 16 years (i.e., 2002-2018). The data analyzed in the present study included age, gender, causative drug, length of hospital stay, received treatment, and complications.

Results: A total of 47 patients, consisting of 21 males and 26 females, were investigated in this study. Out of the 47 patients, 34, 9, and 4 cases were diagnosed with SJS, TEN, and SJS/TEN overlap, respectively. Regarding the etiology of these conditions, 45 cases (95.7\%) were drug-related, while the remaining 2 cases were caused by reasons other than drugs. The most common causative drugs were antimicrobials (36.1\%), followed by antiepileptics (27.6\%) and nonsteroidal anti-inflammatory drug (17\%). Furthermore, there one case caused by reaction to allopurinol, and another case resulted from reactions to rabies vaccine. The remaining cases were either non-drug related or multi-drug related. The most common complications were infections (21.2\%), followed by ocular problems (19.1\%) and gastrointestinal bleeding (2.1\%).

Conclusion: As the findings indicated, antimicrobials were the most common causative drugs of SJS and TEN. The physicians should be aware of the clinical manifestations of SJS and TEN with regard to the severe complications of these conditions, including ocular damages, and their risk of mortality. Moreover, it is required to enhance public awareness regarding the avoidance of self-medication with antimicrobial drugs.

Keywords: Drug Reaction, Stevens-Johnson Syndrome, Toxic Epidermal Necrolysis 


\title{
بررسى سندرم استيونس جانسون و نكروليز إيدر مى سمى در بيماران بسترى در بيمار ستان سيناى همدان: يك مطالعه 19 ساله
}

\author{
"درام عليرضائى'، محمد رضا سبحان'، على سعادتمند ؟؟*"

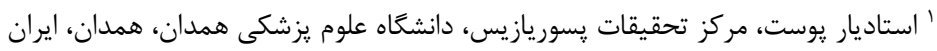

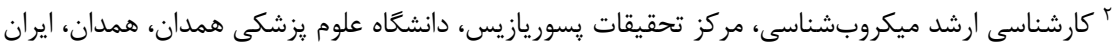 \\ * نويسنده مسئول: على سعادتمند، مركز تحقيقات يسوريازيس، دانشكاه علوم يزشكى همدان، همدان، ايران.
} ايميل:Ali.s_umsha@yahoo.com

جكيده

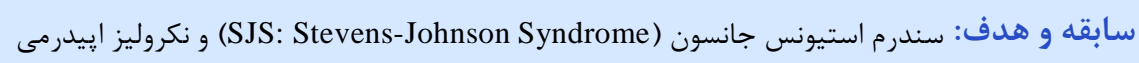
سمى (TEN: Toxic Epidermal Necrolysis) از جمله واكنش هاى شديد و و بالقوه تهديدكننده حيات

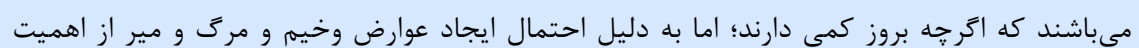

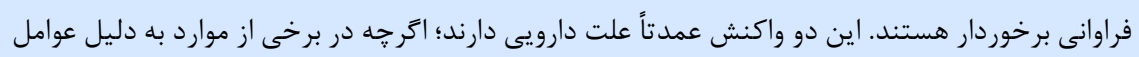

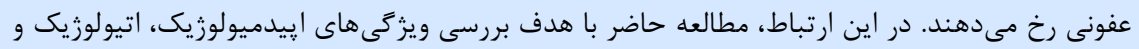

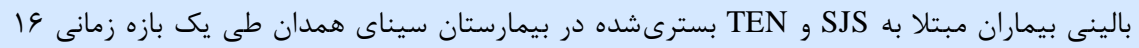
ساله انجام شد. باليني بيمارن

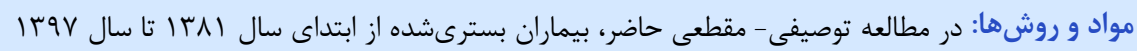

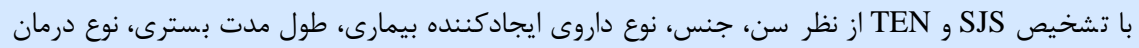
و عوارض ايجادشده بررسى ترديدند.

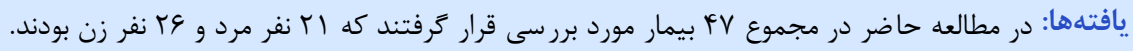

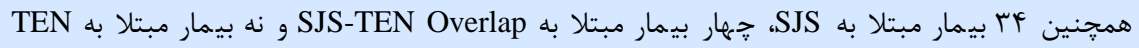

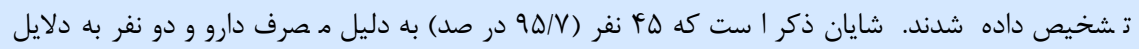

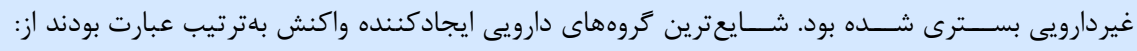

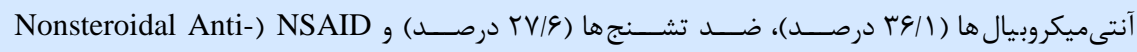

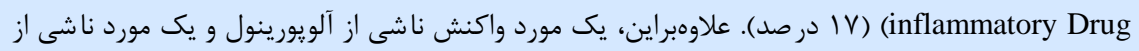

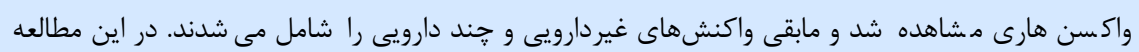

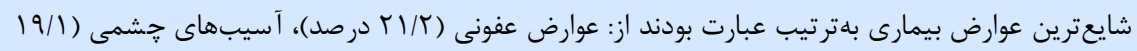

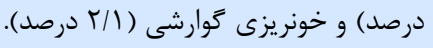

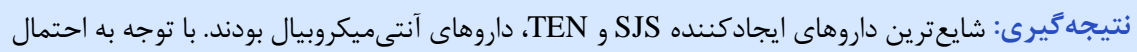

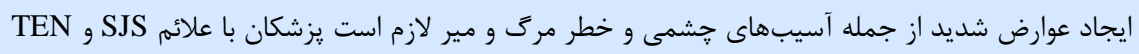

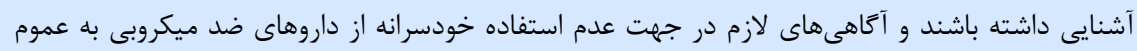

$$
\text { مردم ارائه گردد. }
$$

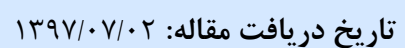

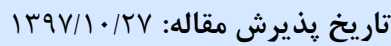

تمامى حقوق نشر براى دانشكاه علوم يزشكى همدان محفوظ است.

وازَّان كليدى: سندرم استيونس جانسون، نكروليز إيدرمى سمى، واكنش دارويى

واكنشها ناگمهانى بوده و علائمى مانند تب، گلودرد و احساس

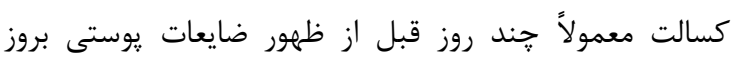

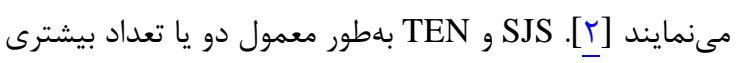

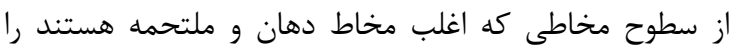

سندرم استيونس جانسون (SJS) و فرم گستردهتر آن،

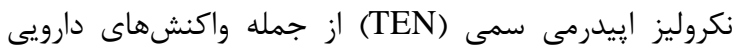

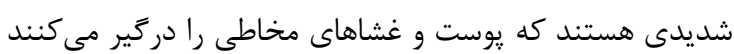

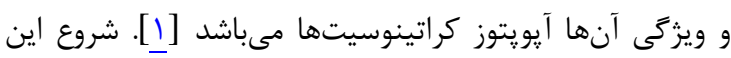


مطالعه ميانكين فاصله زمانى بين مصرف دارو و شروع علائم

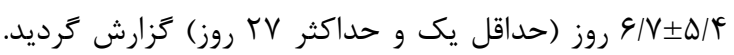

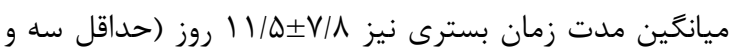

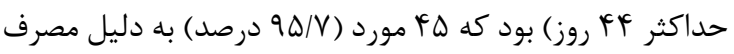

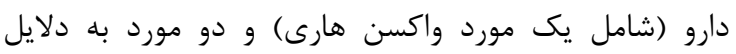

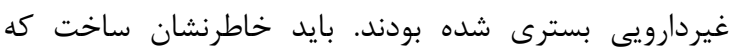

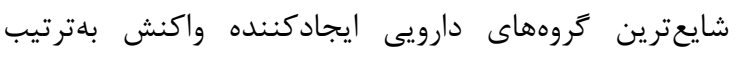

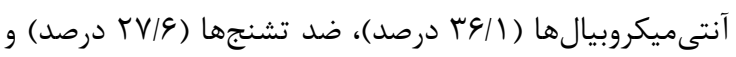

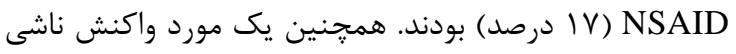
از آلويورينول و يك مورد ناشى از واكسن هارى مشاهده كردين مانديد

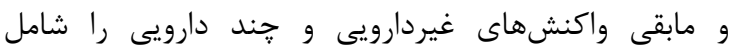

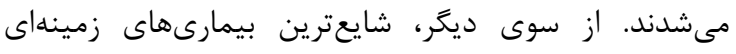

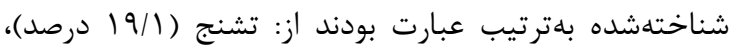

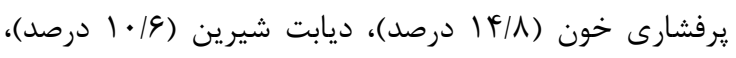

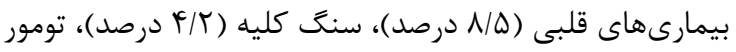

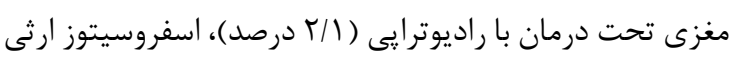

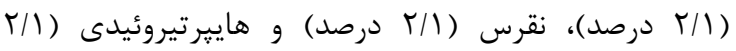

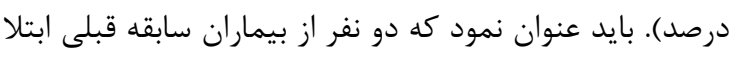

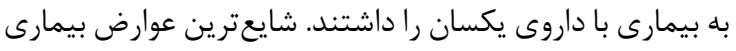

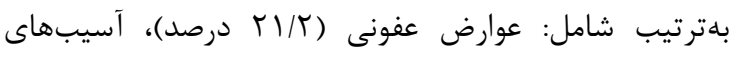

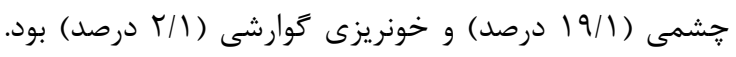

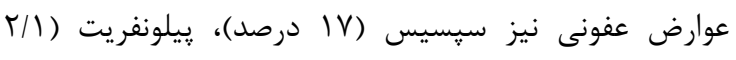

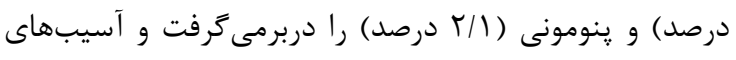

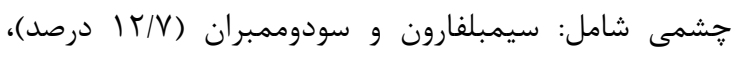

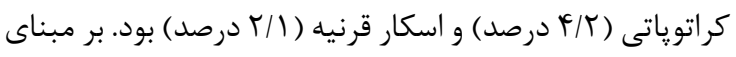

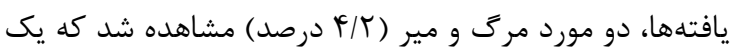

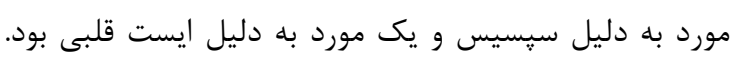

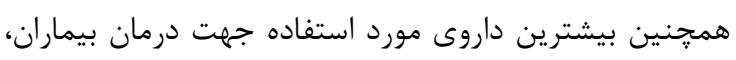

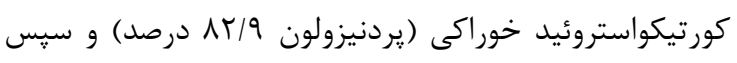

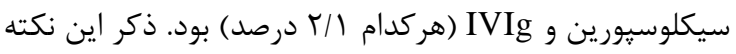

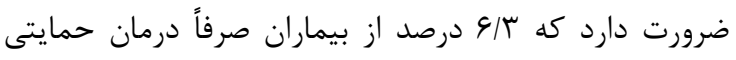

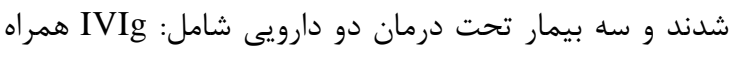

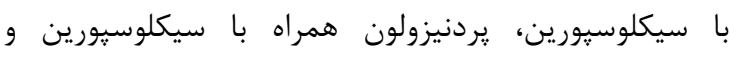

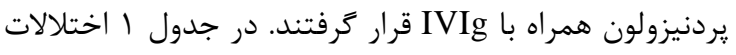

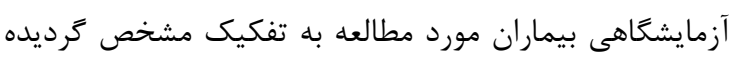

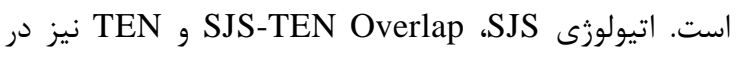
جدول r قابل مشاهده مىباشد. لازم به ذكر است كه در اين مطالعه يك مورد SJS ناشى مداند از مصرف آموكسىسيلين به همراه كليندامايسين، يك اسن مورد

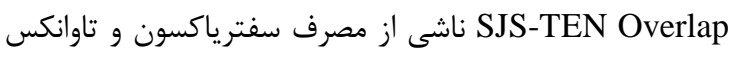

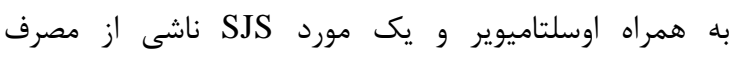

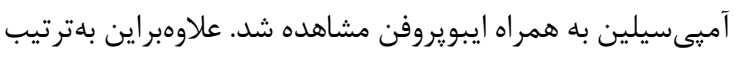

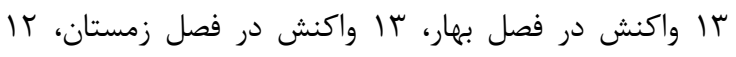

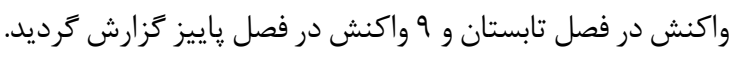

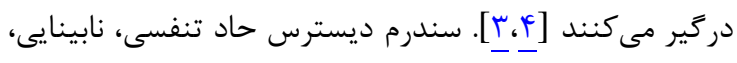

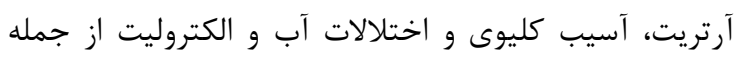

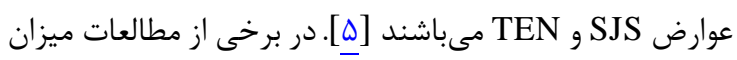

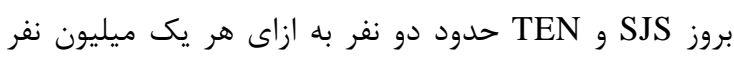

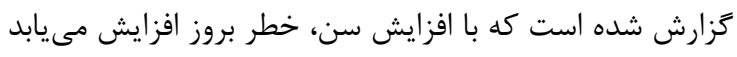

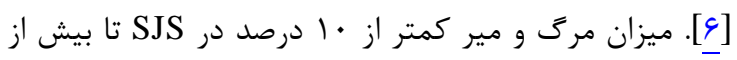

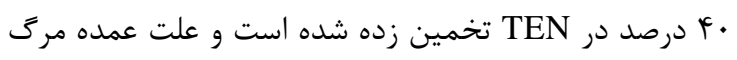

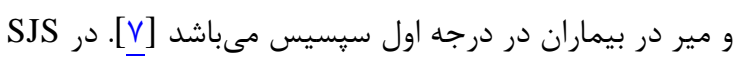

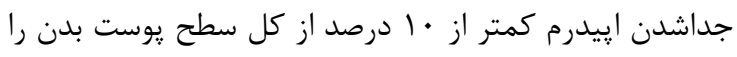

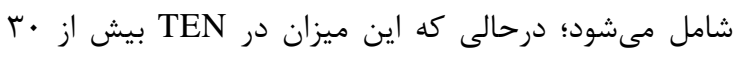

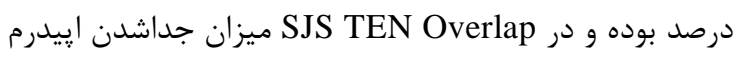

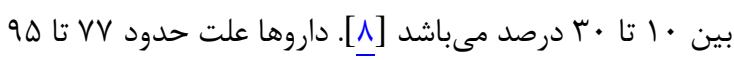

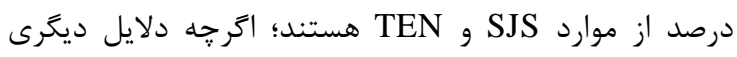
همجون عفونت دستكاه تنفسى با مايكويلاسما نيز بله إعنوان

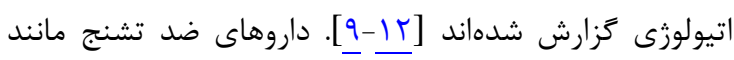

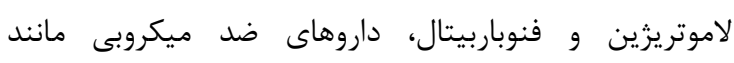

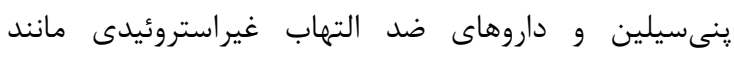

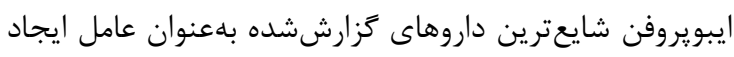

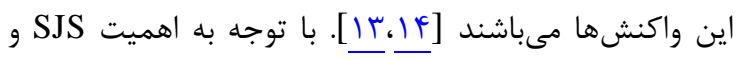

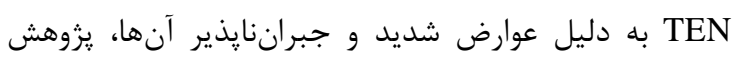

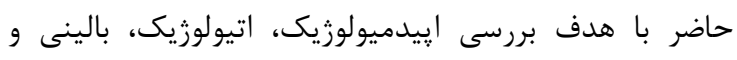

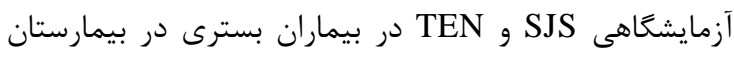

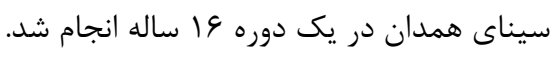

\section{مواد و روشها}

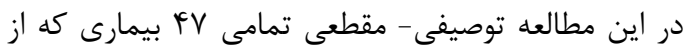

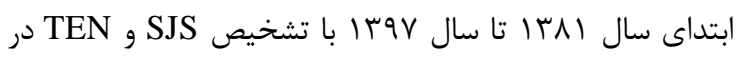

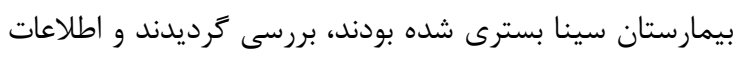

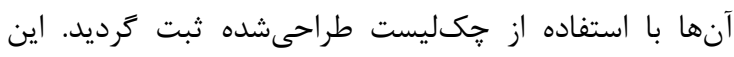

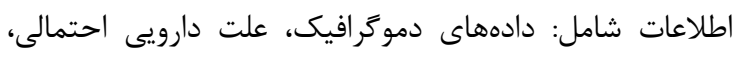

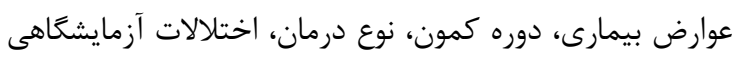

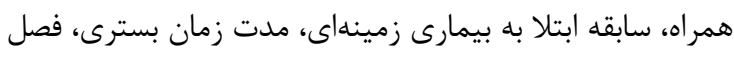

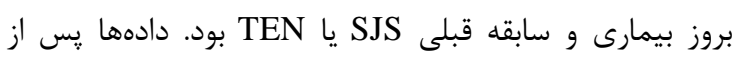

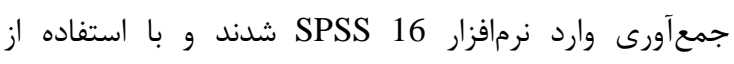
شاخصهاى آمار توصيفى تجزيه و تحليل كرديدند.

يافته ها

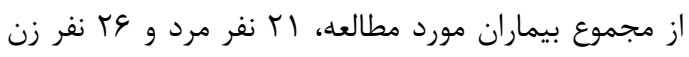

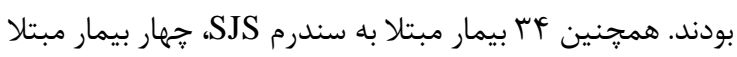

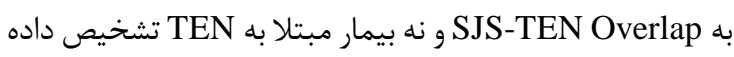

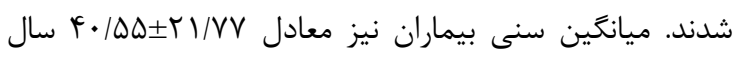

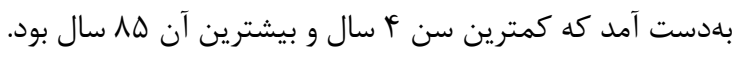

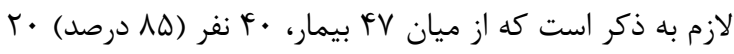

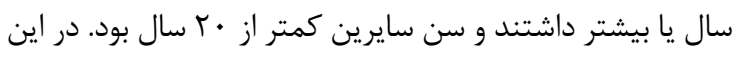


جدول ا: اختلالات آزمايشعاهى در بيماران مبتلا به SJS، و SJS-TEN Overlap

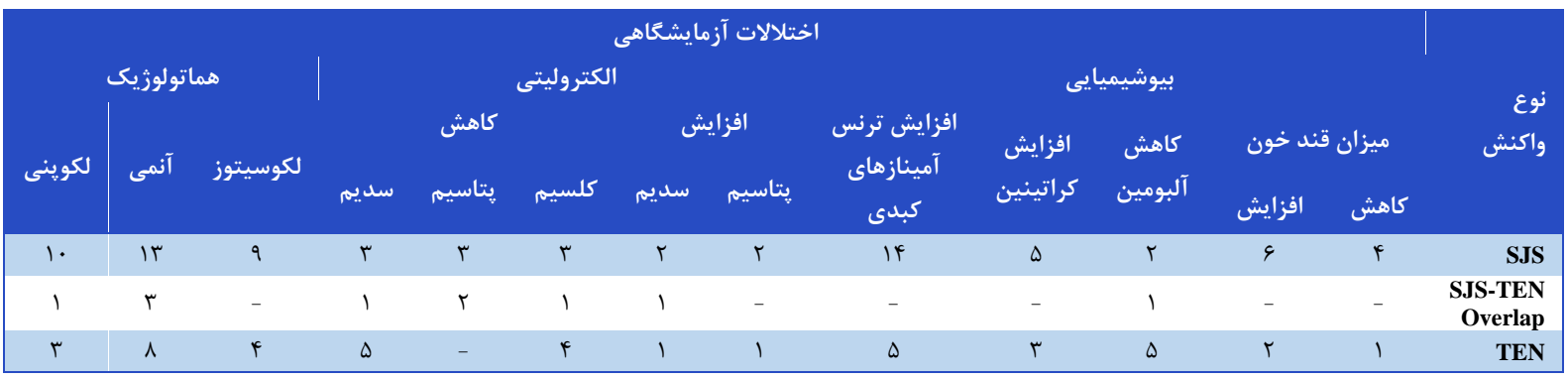

جدول r: اتيولوزى واكنش در بيماران مبتلا به SJS-TEN Overlap و TSS

\begin{tabular}{|c|c|c|c|}
\hline TEN & $\begin{array}{c}\text { نوع واكنش } \\
\text { SJS-TEN Overlap }\end{array}$ & SJS & اتيولوزى \\
\hline- & - & $\Delta$ & ينى پيلين \\
\hline- & 1 & 1 & سفتر ياكسون \\
\hline r & - & - & ايمى ينم \\
\hline 1 & - & 1 & كوتريموكسازول \\
\hline- & - & 1 & جنتامايسين \\
\hline 1 & - & 1 & سييروفلوكساسين \\
\hline- & - & 1 & آموكسىسيلين \\
\hline- & - & 1 & آسيكلووير \\
\hline 1 & - & $\Delta$ & لاموتريزين \\
\hline 1 & - & 1 & كاربامازِين \\
\hline- & - & r & فنوباربيتال \\
\hline- & - & r & فنى توئين \\
\hline- & - & r & شيروكسيكام \\
\hline 1 & 1 & - & ايبويروفن \\
\hline- & - & r & سلكوكسيب \\
\hline- & - & r & نايروكسن \\
\hline 1 & - & - & مفناميك اسيد \\
\hline- & 1 & 1 & آلويورينول \\
\hline 1 & - & - & واكسن هارى \\
\hline- & - & r & غيردارويى \\
\hline
\end{tabular}

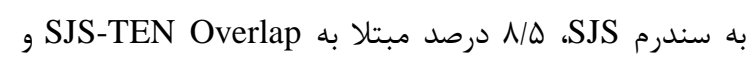

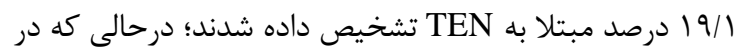

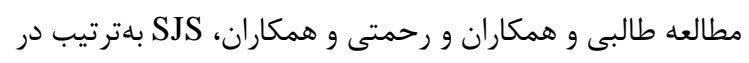

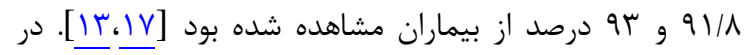

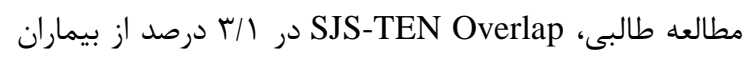

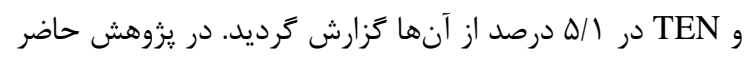

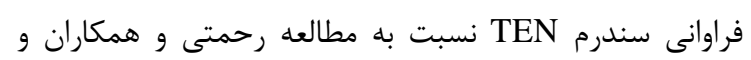

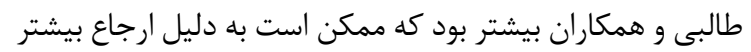

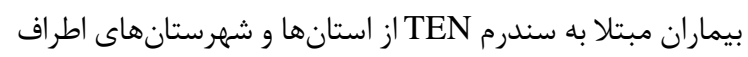
به مركز استان به دليل كمبود امكانات يزشكى و درمانى باشد.

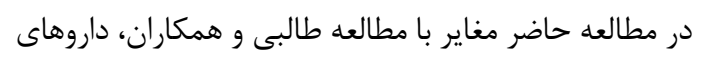

در مطالعه حاضر به بررسى علل بروز سندرم SJS و TEN

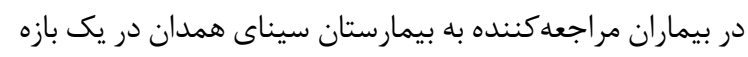

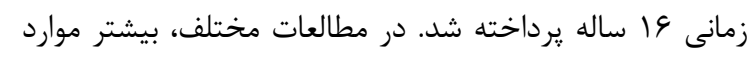

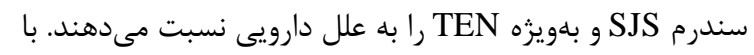

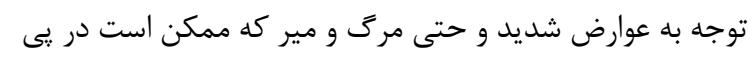

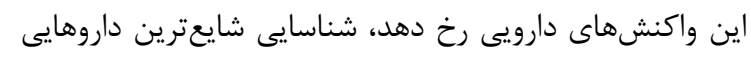

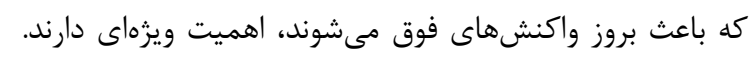

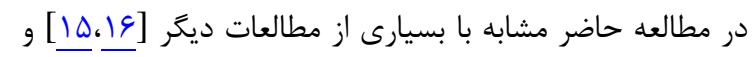

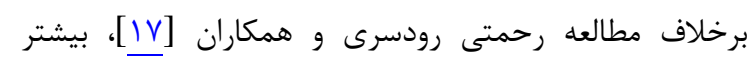
مبتلايان را بانوان تشكيل مىدادند. رحمني رودسري

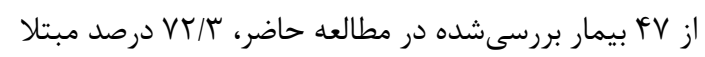


ذكر اين نكته ضرورت دارد كه درمان ضايعات يوستى بيمار مشابه با درمان سوختكى مىباشد؛ اگرجه تفاوتهايى نيز وجود دارد؛

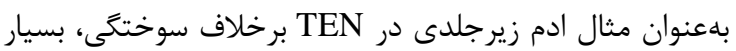

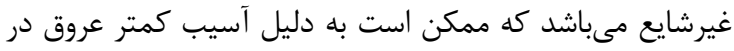

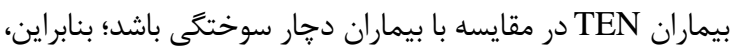

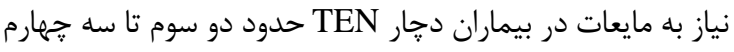

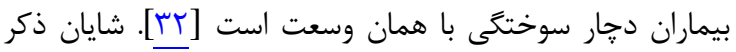

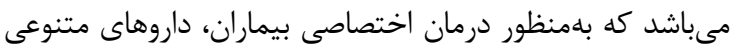

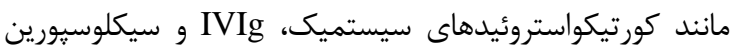
توصيه شده است (بيشتر بيماران در مطالعه حاضر تحت درمان بان

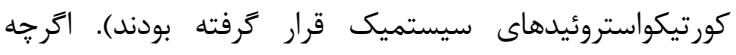
مطالعات متعددى در ارتباط با سودمندى كورتيكواستروئيدها وجود دارد؛ اما برخى از مطالعات بيان مى كنند كه با تجويز اين كروهي

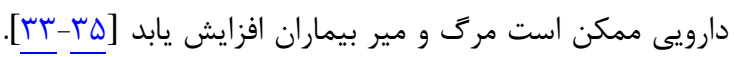

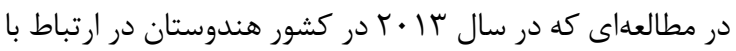

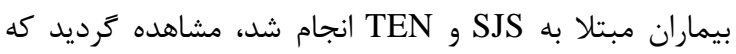

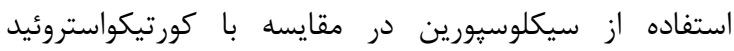

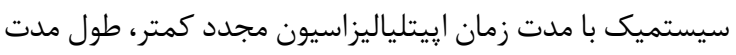

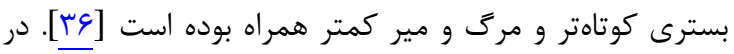

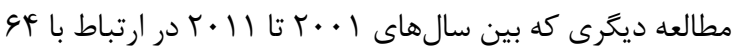

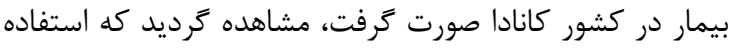

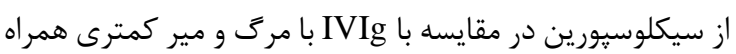

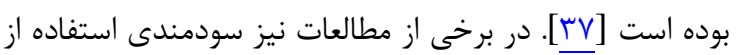

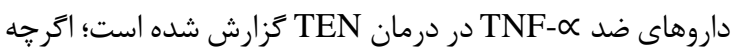
بيشتر اين مطالعات حجم نمونه بسيار كمى داشته و فاقد كرون

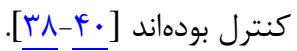

\section{نتيجه تيرى}

در مطالعه حاضر مشابه با بسيارى از مطالعات ديخر،

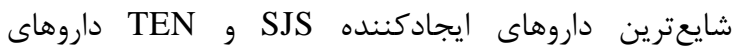

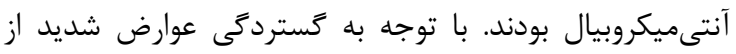

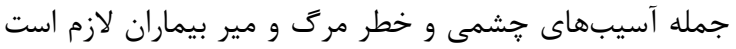

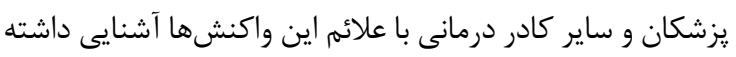

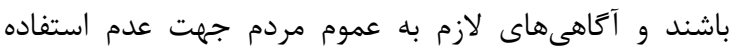
خودسرانه از داروهاى ضد ميكروبى ارائه تردد.

\section{تشكر و قدرداذى}

مقاله حاضر بركرفته از طرح تحقيقاتى مصوب دانشعاه علوم

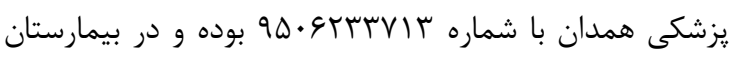

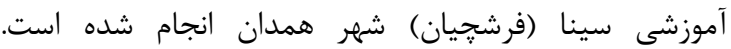

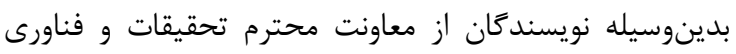
دانشعاه و تمامى افرادى كه در مراحل مختلف مطالعه صميمانه

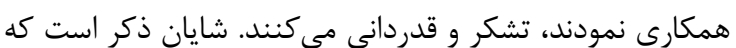

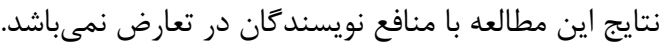

آنتى ميكروبيال شايعترين داروهاى ايجادكننده SJS و TEN

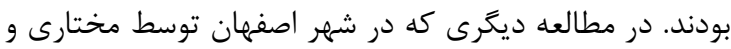

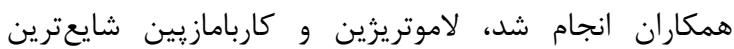

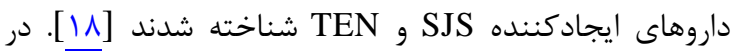
مطالعات متعددى ثابت شده است كه فاكتورهاى زنتيكى بلدويزه

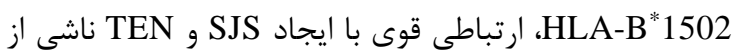

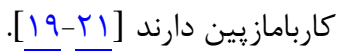
در اين راستا در يزوهشى كه توسط وانتَ و همكاران در سال

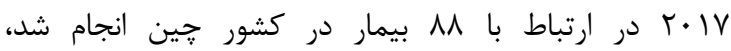

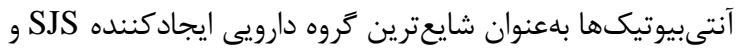
TEN

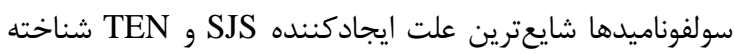

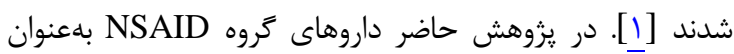

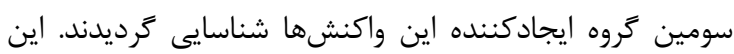

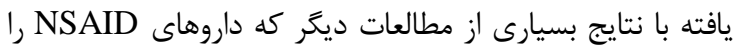

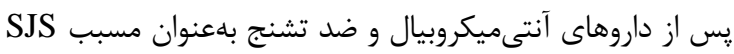

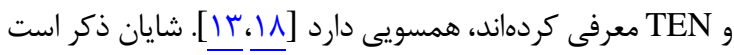
كه در مطالعه حاضر يك مورد SJS-TEN Overlap به دليل مصرف آلويورينول مشاهده گرديد؛ درحالى كه در يك مطاصن مطالعه

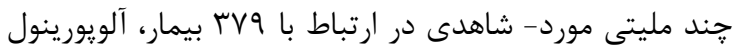

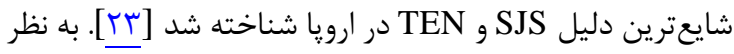
مىرسد كه استعداد ابتلا به SJS و TEN بر اثر اثر مصرف آلويورينول با برخى از فاكتورهاى زنتيكى از جمله

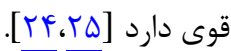
از سوى ديخر، در مطالعه حاضر يك مورد TEN برد إنب اثر تزريق

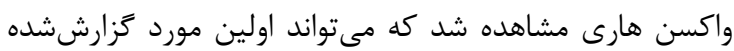
دEN

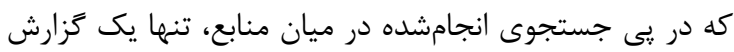

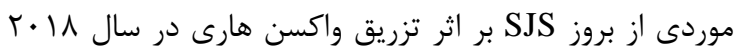
يافت شد [ب مورد. در مطالعه حاضر شايعترين بيمارى زمينهاى مشاهدهشده در

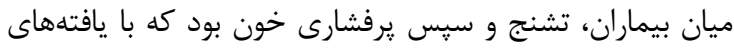

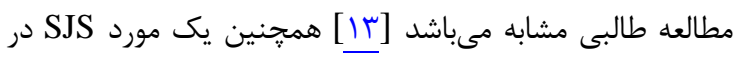

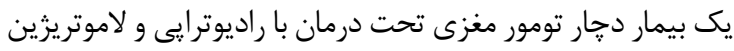

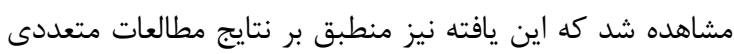

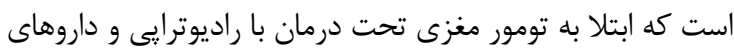

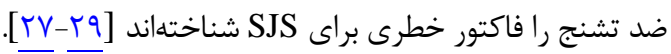
جهت درمان TEN و SJS ابتدا بايد تمام داروهايى كه ممكن

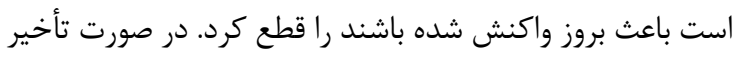

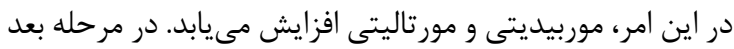

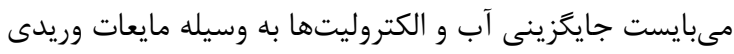

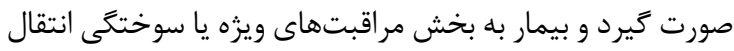

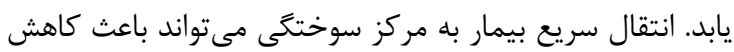

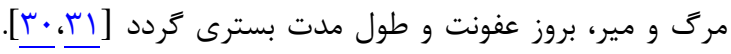




\section{REFERENCES}

1. Letko E, Papaliodis DN, Papaliodis GN, Daoud YJ, Ahmed AR, Foster CS. Stevens-Johnson syndrome and toxic epidermal necrolysis: a review of the literature. Ann Allergy Asthma Immunol. 2005;94(4):419-36. PMID: 15875523 DOI: 10.1016/S1081-1206(10)61112-X

2. Schneider JA, Cohen PR. Stevens-Johnson syndrome and toxic epidermal necrolysis: a concise review with a comprehensive summary of therapeutic interventions emphasizing supportive measures. Adv Ther. 2017;34(6):1235-44. PMID: 28439852 DOI: 10.1007/s12325-017-0530-y

3. Bouvresse S, Valeyrie-Allanore L, Ortonne N, Konstantinou MP, Kardaun SH, Bagot M, et al. Toxic epidermal necrolysis, DRESS, AGEP: do overlap cases exist? Orphanet J Rare Dis. 2012;7:72. PMID: 23009177 DOI: 10.1186/1750-1172-7-72

4. Choon SE, Lai NM. An epidemiological and clinical analysis of cutaneous adverse drug reactions seen in a tertiary hospital in Johor, Malaysia. Indian J Dermatol Venereol Leprol. 2012;78(6):734-9. PMID: 23075643 DOI: 10.4103/03786323.102367

5. Saeed H, Mantagos IS, Chodosh J. Complications of Stevens-Johnson syndrome beyond the eye and skin. Burns. 2016;42(1):20-27. PMID: 25865527 DOI: 10.1016/j. burns.2015.03.012

6. Rzany B, Mockenhaupt M, Baur S, Schröder W, Stocker U, Mueller J, et al. Epidemiology of erythema exsudativum multiforme majus, Stevens-Johnson syndrome, and toxic epidermal necrolysis in Germany (1990-1992): structure and results of a population-based registry. J Clin Epidemiol. 1996;49(7):769-73. PMID: 8691227

7. Hosaka H, Ohtoshi S, Nakada T, Iijima M. Erythema multiforme, Stevens-Johnson syndrome and toxic epidermal necrolysis: frozen-section diagnosis. J Dermatol. 2010; 37(5):407-12. PMID: 20536645 DOI: 10.1111/j.13468138.2009.00746.X

8. Patel T, Barvaliya M, Sharma D, Tripathi C. A systematic review of the drug-induced Stevens-Johnson syndrome and toxic epidermal necrolysis in Indian population. Indian $J$ Dermatol Venereol Leprol. 2013;79(3):389-98. PMID: 23619444 DOI: 10.4103/0378-6323.110749

9. Sehgal VN, Srivastava G. Toxic epidermal necrolysis (TEN) Lyell's syndrome. J Dermatolog Treat. 2005;16(5-6):278-86. PMID: 16428146 DOI: 10.1080/09546630500375684

10. Roujeau JC, Stern RS. Severe adverse cutaneous reactions to drugs. N Engl J Med. 1994;331(19):1272-85. PMID: 7794310 DOI: 10.1056/NEJM199411103311906

11. Fournier S, Bastuji-Garin S, Mentec H, Revuz J, Roujeau JC. Toxic epidermal necrolysis associated with Mycoplasma pneumoniae infection. Eur J Clin Microbiol Infect Dis. 1995;14(6):558-9. PMID: 7588837

12. McDermott AJ, Taylor BM, Bernstein KM. Toxic epidermal necrolysis from suspected mycoplasma pneumoniae infection. Mil Med. 2013;178(9):e1048-50. PMID: 24005558 DOI: 10.7205/MILMED-D-13-00139

13. Talebi R, Saki N, Raeisi Shahraki H, Owji SH. An epidemiological study of Stevens-Johnson syndrome and toxic epidermal necrolysis during 2010-2015 at Shahid Faghihi Hospital, Shiraz, Iran. Iran J Med Sci. 2018; 43(4):421-5. PMID: 30046212

14. Lerch M, Mainetti C, Terziroli Beretta-Piccoli B, Harr T. Current perspectives on Stevens-Johnson syndrome and toxic epidermal necrolysis. Clin Rev Allergy Immunol. 2018;54(1):147-76. PMID: 29188475 DOI: $10.1007 / s 12016-$ 017-8654-Z

15. Yamane Y, Matsukura S, Watanabe Y, Yamaguchi Y, Nakamura K, Kambara T, et al. Retrospective analysis of Stevens-Johnson syndrome and toxic epidermal necrolysis in 87 Japanese patients--Treatment and outcome. Allergol Int. 2016;65(1):74-81. PMID: 26666483 DOI: 10.1016/j.alit. 2015.09.001

16. Arantes LB, Reis CS, Novaes AG, Carvalho MR, Gottems LB, Novaes M. Stevens-Johnson syndrome and toxic epidermal necrolysis: epidemiological and clinical outcomes analysis in public hospitals. An Bras Dermatol. 2017;92(5):661-7. PMID: 29166503 DOI: 10.1590/abd18064841.20176610
17. Rahmati Roodsari M, Gorgzadeh L, Zali H. Cause, clinical course and complications in patients with Stevens- Johnson syndrome and Toxic Epidermal Necrolysis. Res Med. 2013;36(5):66-71. [Persian]

18. Mokhtari F, Nikyar Z, Naeini BA, Esfahani AA, Rahmani S. Adverse cutaneous drug reactions: eight year assessment in hospitalized patients. J Res Med Sci. 2014;19(8):720-5. PMID: 25422656

19. Tangamornsuksan W, Chaiyakunapruk N, Somkrua R, Lohitnavy M, Tassaneeyakul W. Relationship between the HLA-B*1502 allele and carbamazepine-induced StevensJohnson syndrome and toxic epidermal necrolysis: a systematic review and meta-analysis. JAMA Dermatol. 2013;149(9):1025-32. PMID: 23884208 DOI: 10.1001/ jamadermatol.2013.4114

20. Zhang Y, Wang J, Zhao LM, Peng W, Shen GQ, Xue L, et al. Strong association between HLA-B* 1502 and carbamazepine-induced Stevens-Johnson syndrome and toxic epidermal necrolysis in mainland Han Chinese patients. Eur J Clin Pharmacol. 2011;67(9):885-7. PMID: 21424386 DOI: 10.1007/s00228-011-1009-4

21. Chen P, Lin JJ, Lu CS, Ong CT, Hsieh PF, Yang CC, et al. Carbamazepine-induced toxic effects and HLA-B* 1502 screening in Taiwan. $N$ Engl J Med. 2011;364(12):1126-33. PMID: 21428768 DOI: 10.1056/NEJMoa1009717

22. Wang L, Mei XL. Retrospective analysis of Stevens-Johnson syndrome and toxic epidermal necrolysis in 88 Chinese patients. Chin Med J. 2017;130(9):1062-8. PMID: 28469101 DOI: 10.4103/0366-6999.204929

23. Halevy S, Ghislain PD, Mockenhaupt M, Fagot JP, Bouwes Bavinck JN, Sidoroff A, et al. Allopurinol is the most common cause of Stevens-Johnson syndrome and toxic epidermal necrolysis in Europe and Israel. J Am Acad Dermatol. 2008;58(1):25-32. PMID: 17919772 DOI: 10.1016/j.jaad.2007.08.036

24. Hung SI, Chung WH, Liou LB, Chu CC, Lin M, Huang HP, et al. HLA-B* 5801 allele as a genetic marker for severe cutaneous adverse reactions caused by allopurinol. Proc Natl Acad Sci U S A. 2005;102(11):4134-9. PMID: 15743917 DOI: 10.1073/pnas.0409500102

25. Somkrua R, Eickman EE, Saokaew S, Lohitnavy M, Chaiyakunapruk N. Association of HLA-B* 5801 allele and allopurinol-induced Stevens Johnson syndrome and toxic epidermal necrolysis: a systematic review and meta-analysis. BMC Med Genet. 2011;12:118. PMID: 21906289 DOI: 10.1186/1471-2350-12-118

26. Ma L, Du X, Dong Y, Peng L, Han X, Lyu J, et al. First case of Stevens-Johnson syndrome after rabies vaccination. $\mathrm{Br} \mathrm{J}$ Clin Pharmacol. 2018;84(4):803-5. PMID: 29333656 DOI: 10.1111/bcp.13512

27. Khafaga YM, Jamshed A, Allam AA, A. Mourad W, Al Eisa A, Ezzat A, et al. Stevens-Johnson syndrome in patients on phenytoin and cranial radiotherapy. Acta Oncologica. 1999;38(1):111-6. PMID: 10090698

28. Eralp Y, Aydiner A, Tas F, Saip P, Topuz E. Stevens-Johnson syndrome in a patient receiving anticonvulsant therapy during cranial irradiation. Am J Clin Oncol. 2001;24(4):34750. PMID: 11474258

29. Oner Dincbas F, Yörük S, Demirkesen C, Uzel O, Koca S. Toxic epidermal necrolysis after cranial radiotherapy and phenytoin treatment. Onkologie. 2004;27(4):389-92. PMID: 15347896 DOI: $10.1159 / 000079094$

30. Murphy JT, Purdue GF, Hunt JL. Toxic epidermal necrolysis. J Burn Care Rehabil. 2002;23(2):87-96. PMID: 9313122

31. McGee T, Munster A. Toxic epidermal necrolysis syndrome: mortality rate reduced with early referral to regional burn center. Plast Reconstr Surg. 1998;102(4):1018-22. PMID: 9734418

32. Ghislain PD, Roujeau JC. Treatment of severe drug reactions: Stevens-Johnson syndrome, toxic epidermal necrolysis and hypersensitivity syndrome. Dermatol Online J. 2002;8(1):5. PMID: 12165215

33. Tegelberg-Stassen MJ, van Vloten WA, Baart de la Faille $\mathrm{H}$. Management of nonstaphylococcal toxic epidermal necrolysis: follow-up study of 16 case histories. 
Dermatologica. 1990;180(3):124-9. PMID: 2187719

34. Halebian PH, Corder VJ, Madden MR, Finklestein JL, Shires GT. Improved burn center survival of patients with toxic epidermal necrolysis managed without corticosteroids. Ann Surg. 1986;204(5):503-12. PMID: 3767483

35. Cheriyan S, Patterson R, Greenberger PA, Grammer LC, Latall J. The outcome of Stevens-Johnson syndrome treated with corticosteroids. Allergy Proc. 1995;16(4):151-5. PMID: 8566720

36. Singh G, Chatterjee M, Verma R. Cyclosporine in Stevens Johnson syndrome and toxic epidermal necrolysis and retrospective comparison with systemic corticosteroid. Indian J Dermatol Venereol Leprol. 2013;79(5):686-92. PMID: 23974585 DOI: 10.4103/0378-6323.116738

37. Kirchhof MG, Miliszewski MA, Sikora S, Papp A, Dutz JP. Retrospective review of Stevens-Johnson syndrome/toxic epidermal necrolysis treatment comparing intravenous immunoglobulin with cyclosporine. J Am Acad Dermatol. 2014;71(5):941-7. PMID: 25087214 DOI: 10.1016/j. jaad.2014.07.016

38. Zarate-Correa L, Carrillo-Gomez D, Ramirez-Escobar A, Serrano-Reyes C. Toxic epidermal necrolysis successfully treated with infliximab. Case Rep Crit Care. 2017;23(1):613. PMID: 23653980

39. Scott-Lang V, Tidman M, McKay D. Toxic epidermal necrolysis in a child successfully treated with infliximab. Pediatr Dermatol. 2014;31(4):532-4. PMID: 23072342 DOI: 10.1111/pde.12029

40. Wojtkiewicz A, Wysocki M, Fortuna J, Chrupek M, Matczuk M, Koltan A. Beneficial and rapid effect of infliximab on the course of toxic epidermal necrolysis. Acta Derm Venereol. 2008;88(4):420-1. PMID: 18709327 DOI: 10.2340/00015555$\underline{0462}$ 